\title{
Sex-specific costs of hatching last: an experimental study on herring gulls (Larus argentatus)
}

\author{
Maria I. Bogdanova $\cdot$ Ruedi G. Nager
}

Received: 21 November 2007 /Revised: 22 February 2008 / Accepted: 13 March 2008 / Published online: 15 April 2008

(C) Springer-Verlag 2008

\begin{abstract}
An organism's pattern of development can have important long-term fitness effects. In species where the sexes differ in size or other phenotypic traits, they may also have different optimal developmental rates. This influences both parental sex allocation strategies and susceptibility of the sexes to early developmental conditions. However, sex differences in developmental rate and vulnerability to environment during the embryonic period are not well understood. In birds, sibling competition and hatching asynchrony may select for accelerated embryonic development of the last offspring in order to reduce their competitive disadvantage after hatching. They may advance their hatching in response to vocal stimuli by the older siblings. It is, however, unclear whether this flexibility in developmental rates is sex specific. In this study, we experimentally manipulated between-embryo contact and tested whether this affected the pre-natal developmental rate and post-hatching performance of male and female offspring from last-laid eggs in the herring gull. Post-hatching performance was measured both in competitive and noncompetitive situations. Among young incubated in isolation, males hatched faster than females, but both sexes fledged in similar, relatively good condition. Among young incubated with normal between-embryo contact, hatching time did not differ between sexes, but males fledged in poorer condition than females, regardless of whether they were reared singly or in a brood. These results suggest that
\end{abstract}

Communicated by J. Graves

M. I. Bogdanova $(\bowtie) \cdot$ R. G. Nager

Division of Environmental and Evolutionary Biology,

Institute of Biomedical and Life Sciences, University of Glasgow,

Graham Kerr Building,

Glasgow G12 8QQ, UK

e-mail: M.Bogdanova@bio.gla.ac.uk male and female offspring differ in their ability to mitigate the costs of hatching asynchrony.

Keywords Embryo development · Sex effects .

Hatching asynchrony $\cdot$ Sibling competition

\section{Introduction}

Developmental patterns can vary considerably both within and between species and can have long-lasting consequences for organisms' performance later in life (Arendt 1997; Lindström 1999; Metcalfe and Monaghan 2001). In species where the sexes differ in adult size or other phenotypic traits, the optimal developmental modes under any given conditions often differ between males and females (Badyaev 2002). This has important implications for parental resource allocation strategies in relation to offspring sex and for susceptibility of the sexes to early developmental conditions. Most evidence for this has been provided by studies focusing on post-natal development. Males have often been shown to be larger and more costly for the parents to raise and thus more vulnerable to unfavourable rearing conditions than females. This is possibly due to traits such as higher metabolic rate, nutrient requirements or androgen levels (e.g. Clutton-Brock 1991; Griffiths 1992; Sheldon et al. 1998). In contrast, sex differences in developmental rate and susceptibility to unfavourable conditions during the embryonic stage have received relatively little attention, even though evidence suggests that such differences exist. For instance, the sexes can differ in size already at hatching irrespective of the size of the eggs from which they hatch (e.g. Rutkowska and Cichoń 2002; Bogdanova et al. 2007). Furthermore, previous studies have shown that male and female embryo 
survival is differentially affected by conditions during embryonic development (Göth and Booth 2005; Pérez et al. 2006), and unfavourable pre-natal developmental conditions can have long-lasting sex-specific effects on future performance (Gorman and Nager 2004).

In species with more than one young in the nest, sibling competition influences offspring survival and developmental rate (Mock and Parker 1997; Schew and Ricklefs 1998). The dynamics of sibling competition can be influenced by differential allocation of resources among the young and differences in age among interacting siblings (Mock and Parker 1997; Lipar and Ketterson 2000; Groothuis et al. 2005). In many birds, siblings hatch asynchronously, resulting in age hierarchies within broods, with junior offspring often suffering reduced competitive ability and poorer survival compared to their older siblings (Mock and Parker 1997; Hillström et al. 2000). The timing of onset of incubation is thought to be the main determinant of hatching asynchrony (Stoleson and Beissinger 1995). However, during the last phase of incubation when the auditory system of the embryos is developed, they often communicate with each other by auditory stimuli (Vince 1969; Brua 2002). These vocalisations may act as a cue about forthcoming competition to later-developing embryos, and there is evidence that they can respond to these cues by accelerating their hatching time, thus reducing their age disadvantage (Vince 1969; Woolf et al. 1976; Persson and Andersson 1999; Muck and Nager 2006). The sexes may differ in their hatching strategy in response to such cues, and this may contribute to sex differences in incubation periods observed in several studies. In the Eurasian kestrel Falco tinnunculus embryos of the larger sex, females, hatch $2 \mathrm{~h}$ sooner than male embryos (Blanco et al. 2003), whereas in the sexually size monomorphic black guillemot Cepphus grylle males hatch 1 day sooner than females (Cook and Monaghan 2004). Other studies, however, either found no sex differences in embryonic periods (Salomons et al. 2006) or contradictory results within the same species (Burke 1992; Dunnington et al. 1993). When sex differences in timing of hatching occur, they can also cause age differences within a brood to be dependent on the sex of the junior chick. Potential asymmetries between offspring of different sex and hatching rank can result in sex-specific within-brood interactions. Such asymmetries in sibling interactions are likely to have an important, but generally neglected, impact on maternal sex allocation strategies (Uller 2006) and can contribute to differences between males and females in their vulnerability to sibling competition.

In this study, we tested experimentally how hatching strategy in response to the social environment at hatching and offspring sex affect offspring performance in herring gulls Larus argentatus. Laridae are sexual size dimorphic where adult males are larger than females (Cramp 1985).
Females commonly lay three eggs, with the third-laid egg typically being smaller than the other two (e.g. Parsons 1970; Nager et al. 2000a). Chicks hatch asynchronously (e.g. Drent 1970; Parsons 1975), and as a result, the junior chick can be disadvantaged compared to its older siblings (e.g. Parsons 1975; Hébert and Barclay 1986). Male gull chicks are also more vulnerable to poor parental condition than females (Nager et al. 1999, 2000b). We experimentally manipulated the last-laid (C-) egg's social environment at hatching by having eggs incubated alone or in contact with other eggs. We then tested whether at hatching male and female embryos responded differently to signals from older nest mates. We further investigated whether the hatching strategy had consequences for C-chick performance during the nestling period, both when competing with nest mates and when reared alone.

\section{Materials and methods}

The data were collected in the spring of 2003 in a large mixed colony of herring gulls and lesser black-backed gulls on Walney Island, Cumbria, UK $\left(54^{\circ} 08^{\prime} \mathrm{N}, 03^{\circ} 16^{\prime} \mathrm{W}\right)$. The modal clutch size of herring gulls in this colony is three eggs (Bogdanova et al. 2006), and only nests with three eggs were included in the following experiment. During laying, nests located in study plots at the centre of the colony were visited daily, and all eggs were marked in the order they were laid (A for the first-laid, B for the secondlaid and $\mathrm{C}$ for the third-laid egg). On the day the first egg was found, nests were randomly assigned to one of two incubation treatments, where between-embryo contact was removed (experimental nests) or not (control nests). In the experimental group, the C-egg was incubated without contact with developing eggs by removing the first two (A- and B-) eggs on the day they were laid. These embryos would have had no information about the presence of older nest mates. The removed eggs were replaced with dummy eggs so that the parents still incubated a clutch of three eggs. On the day the $\mathrm{C}$-egg was laid, it was exchanged with a $\mathrm{C}$-egg from another nest in the same treatment group laid on the same day to control for the potentially confounding correlation between parental egg production effort and postlaying parental care. In the control group, the $\mathrm{C}$-egg was incubated in contact with an A- and a B-egg developing normally. In these nests, the C-embryo would have had information on the presence of more advanced embryos. The C-eggs were exchanged between clutches, as in the experimental group, so that no $\mathrm{C}$-egg was incubated by its biological parents and raised with biological siblings. All $\mathrm{C}$-eggs were weighed to the nearest $0.1 \mathrm{~g}$ using an electronic balance on the day they were laid. C-eggs depredated during incubation $(n=9)$ were excluded from 
the analyses. The nests assigned to the two incubation treatments did not differ in laying date (experimental nests: $n=82$, control nests: $n=80$, Mann-Whitney test: $Z=0.95, P=0.343)$ or in mass of the C-egg ( $t$ test: $t_{160}=$ $1.35, P=0.180)$.

After they hatched in each of the two incubation treatments, half of the C-chicks were reared alone (in a non-competitive brood environment) and half together with nest mates (in a competitive brood environment). This is hereafter referred to as rearing treatment. From half of the nests where $\mathrm{C}$-eggs were incubated together with developing $\mathrm{A}$ - and B-eggs, neonate $\mathrm{A}-$ and B-chicks were transferred into nests where $\mathrm{C}$-eggs were incubated together with the two dummy eggs. Chicks hatching from C-eggs always remained in the same foster nest in which their egg had been incubated. This combination of incubation and rearing treatment created four experimental groups. The transfer of chicks occurred within 1 day of the last chick hatching and none of the fostered chicks were rejected by their foster family. For C-chicks that were raised together with nestmates, the frequency of two or three-chick broods did not differ between the two incubation treatments $(n=31$ and 31, $\chi^{2}=0.41, d f=1, P=0.520$ ).

Since incubation in the herring gull is initiated before the C-egg is laid (Drent 1970), the start of embryonic development for C-eggs was calculated from the day they were laid (day 1 of incubation). The period of pre-hatching development was divided into two parts: between laying and the time when the embryo perforated the egg shell with its beak (external pipping, the laying-to-pipping interval) and between external pipping and hatching (hatching duration). External pipping was recorded on the date when the first clean hole in the eggshell appeared, and hatching was recorded on the date when the chick completely emerged from the shell. Around the expected onset of external pipping (from day 22 of incubation onwards), the study nests were visited twice daily at approximately $12-\mathrm{h}$ intervals to record the pipping and hatching time of the Cegg. C-eggs started pipping on average $25.1 \pm 0.1 \quad(n=131)$ days and hatched $26.2 \pm 0.1$ days $(n=131)$ after being laid. Among the nests where the C-eggs were incubated with between-embryo contact, only those where one or both of the older (A- and B-) siblings hatched were used in the analyses $(n=64,13$ with one older sibling, 51 with two older siblings). At hatching (day 0), each chick was weighed to the nearest $0.1 \mathrm{~g}$; and its head plus bill length, a good descriptor of overall skeletal size in this species (Coulson et al. 1983), was measured to the nearest $0.1 \mathrm{~mm}$. Hatching mass corrected for size (head plus bill length was a covariate in the analysis) was used as an index of body condition at hatching. On the day of hatching, a small blood sample of up to $50 \mu \mathrm{l}$ was taken under licence to determine the sex of the chick using molecular techniques (Griffiths et al. 1998). This gave a total of 131 hatchlings from C-eggs of known sex. After day 3, the body measurements were taken at 4-day intervals until the age of 31 days when chicks were close to fledging. If a chick was not found on the scheduled day and on three following days, it was presumed dead. Growth rates of chicks were calculated for the period of linear (fastest) growth between days 3 and 23 post-hatching as the slope of the linear regression of mass or size on chick age. In the analysis of growth rate, only chicks that survived until the age of 23 days were used $(n=$ 91). Fledgling mass and size were measured when the chicks were 31 days old, and we used fledging mass corrected for size (head plus bill length was a covariate in the analysis) as an index of body condition at fledging.

\section{Data analysis}

Prior to the analyses, assumptions of parametric tests were checked, and where these were not met, appropriate nonparametric tests were used. Duration of laying-to-pipping interval, hatching duration, and post-hatching growth of chicks hatching from $\mathrm{C}$-eggs were analysed using general linear models with treatment and offspring sex as fixed factors and egg mass and laying date as covariates (SPSS 12.0 2003). The analyses of hatching duration and chick growth in addition included laying-to-pipping interval and hatching duration as covariates, respectively. Hatching success of $\mathrm{C}$-eggs and sex ratio and mortality of Cchicks were analysed using generalised linear models (GLM) with binomial error distribution (SAS 8.2 2001). Initially, all explanatory variables and their two-way interactions were tested. The final, simplified model was obtained after sequentially removing the non-significant effects starting from the least significant interaction terms. Interactions are reported only if they were significant $(P<0.05)$. Significance levels for the statistical tests on offspring growth were adjusted using a sequential Bonferroni procedure (Quinn and Keough 2003), as several measures were taken on the same chicks (size and mass at hatching and at fledging, and growth rate). Values are expressed as mean $\pm 1 \mathrm{SE}$, except where nonparametric tests were used.

\section{Results}

Rate of embryonic development and hatching

Eggs started pipping earlier as the season progressed, but there was no effect of incubation treatment or embryo sex on the length of the laying-to-pipping interval [analysis of covariance (ANCOVA) on the laying-to-pipping interval; laying date: $F_{1,129}=16.37, P<0.001$; egg mass: $F_{1,126}=0.23$, 


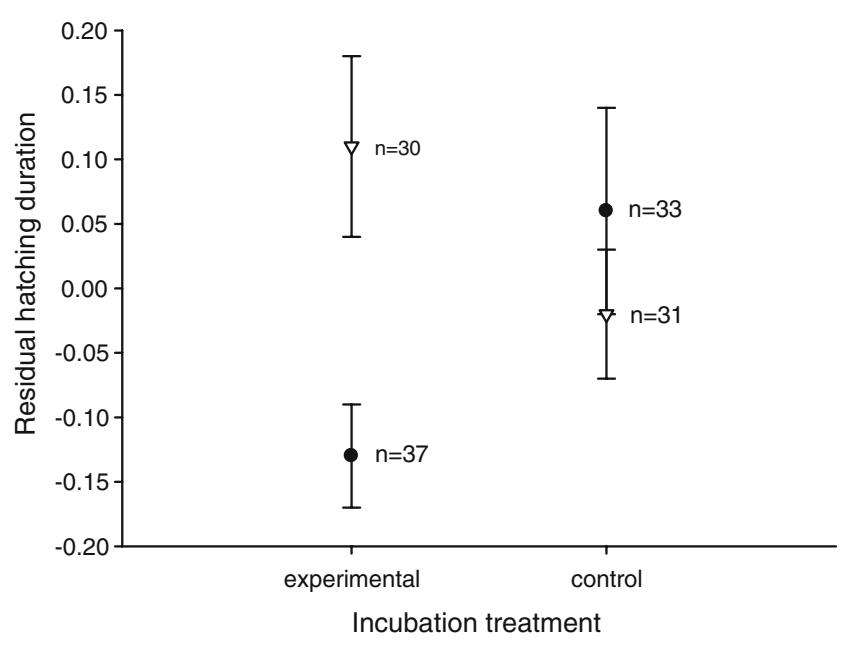

Fig. 1 Mean residual hatching duration $( \pm 1 \mathrm{SE})$ in the two sexes in relation to incubation treatment (experimental $\mathrm{C}$-egg incubated alone, control C-egg in contact with developing embryos, filled circles male, inverted empty triangles female) corrected for laying-to-pipping interval. In experimental nests, males hatched faster than females $\left(n=67, t_{65}=\right.$ $2.90, P=0.005$ ), whereas in control nests, there was no difference in hatching duration between the sexes $\left(n=64, t_{62}=1.00, P=0.320\right)$

$P=0.633$; offspring sex: $F_{1,127}=0.48, P=0.490$; incubation treatment: $\left.F_{1,128}=0.57, P=0.453\right]$. The laying-to-pipping interval shortened by 0.5 days for every 10 days advancement of laying date. Embryos that pipped at an earlier age took longer to hatch, and the effects of the incubation treatment on hatching duration differed between male and female offspring (effect on hatching duration of laying-topipping interval: $F_{1,126}=10.98, P=0.001$; incubation treatment: $F_{1,126}=0.19, P=0.666$; sex: $F_{1,126}=1.77, P=0.186$; treatment $\times$ sex: $F_{1,126}=7.17, P=0.008$; laying date: $F_{1,124}=$ $0.07, P=0.788$; egg mass: $F_{1,125}=0.23, P=0.636$ ). In C-eggs from experimental nests, which had no contact with more advanced siblings, males hatched 0.3 days faster than females. In contrast, in control nests where the C-eggs were incubated in a normal clutch, there was no difference in hatching duration between the sexes: male and female Cembryos hatched on average $26.2 \pm 0.1$ days after the egg was laid (Fig. 1).

Hatching success, hatchling condition and hatchling sex ratio

Smaller C-eggs were less likely to hatch, but incubation treatment did not affect their hatching success (GLM; egg mass: $F_{1,160}=5.33, P=0.022$; incubation treatment: $F_{1,158}=$ $0.20, P=0.653$; laying date: $F_{1,159}=1.19, P=0.276$ ). Hatching success was similar between $\mathrm{C}$-eggs in control $(80 \%$, $n=80)$ and experimental nests $(82 \%, n=82)$. C-eggs that were lighter than the mean had a hatching success of $74 \%$ $(n=84)$, whereas heavier-than-average C-eggs had a hatching success of $89 \%(n=78)$. No young died between pipping and hatching. Offspring sex was not included in the analysis of hatching success because only eggs that hatched were sexed.

Among C-chicks, hatchling size and mass were affected by egg mass but were independent of the incubation treatment, hatching duration and laying date (Table 1). Chicks that hatched from larger eggs were heavier and skeletally larger than chicks that hatched from smaller eggs. Male and female hatchlings had similar body mass, but males were skeletally larger (head plus bill length: $47.1 \pm$ $0.1 \mathrm{~mm}, n=70)$ than females $(46.1 \pm 0.1 \mathrm{~mm}, n=61)$. Hatching mass was independent of hatching size when corrected for egg mass (hatching size: $F_{1,128}=1.49, P=$ 0.225; egg mass: $\left.F_{1,128}=267.80, P<0.001\right)$ and, therefore, considered a good descriptor of hatching condition. Hatching sex ratio of the C-chick was independent of egg mass, laying date or incubation treatment (GLM; egg mass: $F_{1,129}=0.73, P=0.395$; incubation treatment: $F_{1,127}=0.24, P=0.626$; laying date: $F_{1,128}=0.29, P=0.592$ ). There were $52 \%$ males $(n=64)$ in the control incubation treatment and $55 \%$ males $(n=67)$ in the experimental incubation treatment.

\section{Post-hatching chick performance}

After hatching, C-chicks were raised by foster parents either with foster siblings or singly. Where C-chicks were reared together with nest mates, the hatching span between A- and C-chicks in the foster nest did not differ between incubation treatments or male and female $\mathrm{C}$-chicks (2-way non-parametric ANOVA; $n=62$; incubation treatment: $H=$ 1.98, $P=0.159$; sex: $H=0.20, P=0.655$ ).

Rearing treatment and offspring sex, but not incubation treatment, influenced growth rate during the period of

Table 1 Hatching size and mass of chicks from C-eggs in relation to incubation treatment, offspring sex, egg mass, laying date and timing of hatching events $(n=131)$

\begin{tabular}{|c|c|c|c|c|}
\hline & \multicolumn{2}{|l|}{ Hatching size } & \multicolumn{2}{|l|}{ Hatching mass } \\
\hline & $F(d f)$ & $P$ & $F(d f)$ & $P$ \\
\hline Egg mass & $41.68(1,128)$ & $<0.001$ & $372.87(1,129)$ & $<0.001$ \\
\hline Offspring sex & $28.28(1,128)$ & $<0.001$ & $1.45(1,127)$ & 0.231 \\
\hline Laying date & $3.25(1,127)$ & 0.074 & $0.96(1,126)$ & 0.328 \\
\hline Incubation treatment & $1.03(1,126)$ & 0.312 & $0.15(1,125)$ & 0.699 \\
\hline Hatching duration & $0.01(1,125)$ & 0.905 & $1.52(1,128)$ & 0.220 \\
\hline
\end{tabular}

Head plus bill length was taken as a measure of body size. Hatching mass was independent of hatching size when corrected for egg mass (see text) and, therefore, considered a good descriptor of hatching condition.

Statistically significant effects are shown in bold after applying a sequential Bonferroni procedure: $\alpha_{1}=0.025(\alpha / 2), \alpha_{2}=0.05$; the smallest $P$ values were tested at $\alpha_{1}$ and the next at $\alpha_{2}$ (see "Materials and methods"). 
fastest growth. For body mass, C-chicks reared alone grew faster than those reared in a competitive brood environment, and males grew faster than females [ANCOVA; rearing treatment: $F_{1,88}=7.61, P=0.007$; offspring sex: $F_{1,88}=7.50, P=0.007$; egg mass: $F_{1,87}=3.76, P=0.056$; incubation treatment: $F_{1,86}=1.28, P=0.261$; hatching duration: $F_{1,85}=0.47, P=0.495$; laying date: $F_{1,84}=0.17, P=$ 0.680; Table 2 (growth rate)]. Similar results were found for skeletal growth [rearing treatment: $F_{1,88}=4.20, P=0.044$; offspring sex: $F_{1,88}=9.14, P=0.003$; laying date: $F_{1,87}=$ 1.32, $P=0.253$; hatching duration: $F_{1,86}=0.80, P=0.374$; egg mass: $F_{1,85}=0.34, P=0.563$; incubation treatment: $F_{1,84}=0.32, P=0.575$; Table 2 (growth rate)]. For chicks raised in a competitive brood environment, mortality of older nest mates (A- and B-chicks) did not differ between incubation treatments $\left(n=39 ; \chi^{2}=0.42, d f=1, P=0.520\right)$, and nest mate mortality did not affect the growth of $\mathrm{C}$ chicks $(P>0.46)$.

$\mathrm{C}$-chick survival until fledging was marginally affected by the rearing treatment but not by the incubation treatment or offspring sex (GLM; rearing treatment: $F_{1,129}=3.73, P=$ 0.055; hatching duration: $F_{1,124}=0.02, P=0.876$; egg mass: $F_{1,125}=0.07, P=0.798$; sex: $F_{1,126}=0.27, P=0.607$; incubation treatment: $F_{1,127}=0.36, P=0.548$; laying date: $F_{1,128}=$ $1.28, P=0.260$ ). C-chicks reared in a competitive brood environment tended to be less likely to fledge $(55 \%, n=62)$ than chicks reared singly $(71 \%, n=69)$. Given our sample sizes, our test has a power of 0.75 to detect a statistically significant difference in survival of chicks reared singly and in a brood (two-tailed test).

The size of fledglings that hatched from $\mathrm{C}$-eggs was affected by rearing treatment, offspring sex and egg mass (Table 3). C-chicks reared in a competitive brood environment were skeletally smaller at fledging than those reared singly, and males were larger than females [Table 2 (fledging size and mass)]. Interestingly, skeletal size at fledging of C-chicks increased with increasing egg mass. Fledging condition (fledging mass corrected for fledging size) depended on interactions between incubation treatment and offspring sex and between rearing treatment and laying date (Table 3). Early in the season, chicks reared in a competitive brood environment fledged in poorer condition than those reared singly, but the difference between rearing environments disappeared later in the season (Fig. 2). An effect of incubation treatment persisted until fledging, and this was different for male and female chicks. Independent of the rearing treatment, males incubated in control nests fledged in poorer condition than females. There was no difference in fledging condition between males and females incubated in experimental nests (Fig. 3). Further analyses showed that among chicks reared in a competitive brood environment, brood size at fledging did not differ between incubation treatments $(n=34$; Mann-Whitney test: $Z=1.57$, $P=0.116)$ and did not influence fledgling size or condition $(P>0.69)$. However, median brood size at fledging decreased from three chicks early in the season (before the median laying date, 12 May) to two chicks late in the season (after 12 May).

\section{Discussion}

In this study, we tested whether sibling contact during the embryonic stage differentially affects developmental rate of male and female offspring and whether this has consequences for their post-hatching performance. We manipulated the embryo's social environment during incubation and hatching and found a sex-specific effect of this manipulation on hatching duration and fledging condition. Among young incubated in isolation from other developing eggs, males hatched faster than females, but both sexes fledged in similar, relatively good body condition. In

Table 2 Growth rate and fledging size and mass of chicks that hatched from C-eggs in relation to rearing treatment and offspring sex

\begin{tabular}{|c|c|c|c|c|}
\hline & \multicolumn{2}{|c|}{ Reared singly } & \multicolumn{2}{|c|}{ Reared in a brood } \\
\hline & Males & Females & Males & Females \\
\hline \multicolumn{5}{|l|}{ Growth rate } \\
\hline Head plus bill length (mm/day) & $1.95 \pm 0.05$ & $1.79 \pm 0.03$ & $1.84 \pm 0.05$ & $1.69 \pm 0.05$ \\
\hline Body mass (g/day) & $\begin{array}{l}28.18 \pm 1.32 \\
(n=31)\end{array}$ & $\begin{array}{l}24.41 \pm 0.79 \\
(n=21)\end{array}$ & $\begin{array}{l}24.33 \pm 1.53 \\
(n=18)\end{array}$ & $\begin{array}{l}21.15 \pm 1.22 \\
(n=21)\end{array}$ \\
\hline \multicolumn{5}{|l|}{ Fledging size and mass } \\
\hline Head plus bill length (mm) & $101.7 \pm 1.1$ & $95.4 \pm 0.8$ & $97.7 \pm 1.3$ & $93.4 \pm 1.5$ \\
\hline Fledging mass (g) & $\begin{array}{l}787.5 \pm 30.5 \\
(n=29)\end{array}$ & $\begin{array}{l}688.9 \pm 15.2 \\
(n=20)\end{array}$ & $\begin{array}{l}689.1 \pm 36.0 \\
(n=17)\end{array}$ & $\begin{array}{l}632.1 \pm 32.0 \\
(n=17)\end{array}$ \\
\hline
\end{tabular}

Growth rates were calculated over the linear growth phase only for chicks that survived until day 23 (see "Materials and methods", $n=91$ ), Fledgling size (head plus bill length) was measured at day $31(n=83)$. Values are presented as mean \pm 1 SE. 
Table 3 Fledgling size and condition of chicks that hatched from C-eggs in relation to incubation treatment, rearing treatment, offspring sex, egg mass, hatching duration and laying date

\begin{tabular}{|c|c|c|c|c|}
\hline & \multicolumn{2}{|l|}{ Fledgling size } & \multicolumn{2}{|l|}{ Fledgling mass } \\
\hline & $F(d f)$ & $P$ & $F(d f)$ & $P$ \\
\hline Incubation treatment & $0.57(1,77)$ & 0.454 & $5.35(1,75)$ & 0.024 \\
\hline Rearing treatment & $6.57(1,79)$ & 0.012 & $4.66(1,75)$ & 0.034 \\
\hline Offspring sex & $20.88(1,79)$ & $<0.001$ & $9.20(1,75)$ & 0.003 \\
\hline Egg mass & $4.04(1,79)$ & 0.048 & $0.56(1,74)$ & 0.455 \\
\hline Hatching duration & $0.69(1,78)$ & 0.410 & $0.21(1,73)$ & 0.651 \\
\hline Laying date & $0.12(1,76)$ & 0.735 & $3.75(1,75)$ & 0.057 \\
\hline Fledgling size & - & - & $373.91(1,75)$ & $<0.001$ \\
\hline Incubation treatment $\times$ offspring sex & $1.83(1,74)$ & 0.180 & $5.06(1,75)$ & 0.027 \\
\hline Rearing treatment $\times$ laying date & $1.97(1,73)$ & 0.165 & $4.38(1,75)$ & 0.040 \\
\hline
\end{tabular}

Fledgling size and mass were measured at day $31(n=83)$.

Statistically significant effects are shown in bold after applying a sequential Bonferroni procedure: $\alpha_{1}=0.025(\alpha / 2), \alpha_{2}=0.05$; the smallest $P$ values were tested at $\alpha_{1}$ and the next at $\alpha_{2}$ (see "Materials and methods").

contrast, among young in control clutches in contact with other developing embryos, the two sexes took similar time to hatch (due to males delaying their hatching), but males fledged in significantly poorer condition than females.

\section{Embryonic development}

The interval between laying and pipping did not differ between the two incubation treatments or between male and female embryos. This is not surprising as during early stages of development, embryos are unlikely to be able to communicate and thus influence each other's development

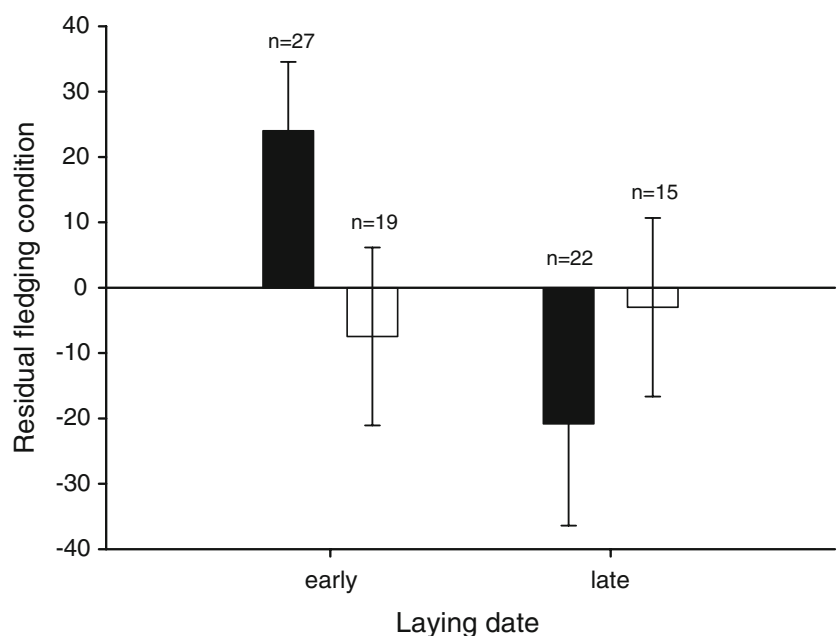

Fig. 2 Chicks that hatched from C-eggs raised singly (filled bars) fledged in better condition than chicks raised together with nest mates (empty bars) early in the season but not late in the season. Early/late refers to whether the eggs were laid before (or on) or after the median laying date (12 May). The statistical analysis was performed using laying date as a continuous variable (Table 3 ). Shown are mean values $\pm 1 \mathrm{SE}$ time (Woolf et al. 1976; Brua 2002) but indicates that incubation performance of control and experimental parents must have been similar. It also suggests that there was no intrinsic difference in developmental rate between the sexes during the earlier stages of incubation. The incubation treatment, however, influenced hatching duration (that is, the interval between pipping and hatching) and its effect was sex specific. In the experimental treatment, where between-embryo contact was eliminated, male embryos hatched faster than females. In the control treatment, where

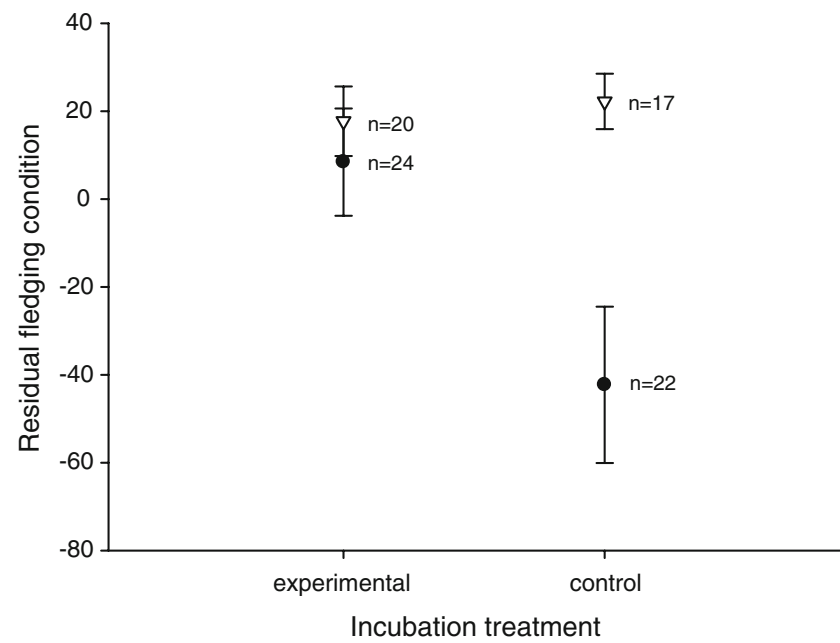

Fig. 3 Mean residual fledging condition (mass corrected for size, $\pm 1 \mathrm{SE}$ ) in relation to offspring sex (filled circles male, inverted filled triangles female) and incubation treatment. Males and females from C-eggs incubated in isolation (experimental treatment) did not differ in fledging condition (sex: $\left.F_{1,41}=0.47, P=0.497\right)$. Among C-chicks incubated in contact with developing eggs (control treatment), males fledged in poorer condition than females (sex: $F_{1,36}=12.09, P=0.001$ ). Numbers in the figure refer to sample sizes 
C-eggs were incubated in contact with two older developing embryos, there was no sex difference in development time. The lack of difference in hatching duration between the sexes in this treatment group was due to males hatching more slowly compared to when they were incubated in isolation. Hatching duration in females was similar in the two treatments.

Phenotypic differences between the sexes (Badyaev 2002) or differences in the hormonal content of eggs containing male and female embryos (Balaban and Hill 1971; Lipar and Ketterson 2000; Eising et al. 2001) may have allowed males to hatch faster than females when incubated in isolation. There are at least two possible explanations for the delayed hatching of males in the presence of older siblings. If communication between embryos acts as a signal of forthcoming competition, the strategy of last-hatching males may be to delay hatching in order to complete their development. Delayed hatching has been shown to result in more complete development of the embryonic neuromuscular system (Nilsson and Persson 2004). This may be beneficial for males hatching last in the brood as it may improve their motor ability and consequently their chances to compete effectively for food, which is of importance given their high growth rate and hence food requirements (e.g. Griffiths 1992). This, however, is unlikely to be the case in our study as the control males actually fledged in poorer condition than females in the same treatment and than both males and females in the experimental treatment. Alternatively, control C-eggs may have experienced lower and more variable incubation temperatures during hatching. In herring gulls, the older siblings can leave the nest cup within hours of hatching with the attending parents then having to divide their attention between the young chicks outside the nest and any eggs still remaining in the nest (Drent 1970; Lee et al. 1993). Indeed, C-eggs of herring gulls experience parental neglect during the pipping to hatching interval, and their incubation temperature is ca. $4^{\circ} \mathrm{C}$ lower than for Aeggs at the same developmental stage (Lee et al. 1993). Ceggs in our experimental clutches would not have been exposed to parental neglect during the last stage of incubation because parents would not have to divide their time between incubating the remaining egg and attending chicks outside the nest. Exposure to sub-optimal temperatures during this critical developmental period can have detrimental effects on offspring performance (Webb 1987; Brua 2002). It is possible that males are vulnerable to such sub-optimal conditions during the final stage of their embryonic development and are, therefore, unable to hatch as fast as they would in a situation where parental neglect is removed. Interestingly, female embryos seem to be less vulnerable to parental neglect, and their hatching duration was not affected. In species with sexual size dimorphism, such as the herring gull, developmental processes and their regulation can differ between males and females and thus result in sex differences in sensitivity to particular developmental stages, with the larger sex (in our case males) commonly being more adversely affected (Badyaev 2002).

\section{Post-hatching performance}

Not surprisingly, larger eggs had higher hatching success, and hatchlings from larger eggs were skeletally larger and heavier than those from smaller eggs (e.g. Williams 1994). As found in previous studies of gulls (e.g. Griffiths 1992; Nager et al. 1999), males were skeletally larger than females already at hatching but were in similar body condition. However, no effect of incubation treatment and hatching duration on the size or mass of offspring of either sex was detected at this stage. This may be because avian embryo growth declines or even stops during the last days of incubation ('plateau phase' in metabolic rate; Vleck and Bucher 1998; Nilsson and Persson 2004), so mass may have reached hatching values already at the time of external pipping.

A number of factors affected offspring performance during the nestling period. Overall, males grew faster and consequently reached on average higher mass and size at fledging compared to females. Faster growth of the larger sex in sexually size-dimorphic species has been reported in a large number of species, including gulls (e.g. CluttonBrock 1991; Griffiths 1992). Also, irrespective of their sex, singly raised chicks grew faster and fledged in better condition than chicks that had to compete with older siblings, but this difference was evident only early in the season. This was because fledgling condition declined with laying date only among singly raised C-chicks but not in Cchicks reared in a brood. As the season progressed, parents raising only one young may have changed their strategy regarding the trade-off between current and future reproduction and reduced their investment in the current reproductive event due to its low potential output. In contrast, pairs raising larger broods late in the season may have a similar parental effort to pairs breeding earlier in the season due to the high perceived success of the current breeding event (Winkler and Allen 1996; Rytkönen 2002; Gasparini et al. 2006). Nestling growth was positively correlated with egg mass. Such relationships between egg mass and offspring performance are often found in birds, although they are usually observed mainly early in the posthatching period and are later overridden by variation in parental provisioning ability (e.g. Christians 2002; Bize et al. 2002; Arnold et al. 2006). However, in our study species $\mathrm{C}$-eggs are generally at the lower end of the range of viable egg sizes (Parsons 1970). Thus, negative effects of small egg size may be difficult to overcome even under the 
benign rearing conditions that singly raised chicks experienced (Nager et al. 2000a).

Most remarkably, the environment in which the young were incubated influenced their condition at fledging. Males incubated in control nests fledged in significantly poorer condition than control females and than experimental males and females. This result was not confounded by the brood size or the hatching asynchrony experienced in the foster nest as these did not differ between incubation treatments and offspring sexes. Hence, the poorer fledgling condition of control males cannot be due to any differences in competitive situation they might have encountered after hatching nor due to sexual differences in competitive ability per se since singly reared control males showed the same poor fledging condition. This suggests that they suffered a developmental cost, possibly due to parental neglect during the final stage of incubation, which was difficult to offset even in a relatively benign rearing environment. Lee et al. (1993) showed that neglect of C-eggs during hatching did not affect egg hatchability or survival of the young during the first week of their life. Similarly, we found no detrimental effects of the incubation treatment on embryo survival or on offspring growth rate during the linear growth phase up to the age of 23 days. However, males from the control incubation treatment were lighter at fledging, implying that they must have put on less mass during the later part of the nestling period compared to the average growth trajectory for this population. This could be possibly related to muscular and/or organ maturation, which occurs in the last days of incubation (Ricklefs and Starck 1998; Nilsson and Persson 2004). Even relatively small effects of unfavourable conditions during early development can have profound and sex-specific long-term consequences for offspring performance (Metcalfe and Monaghan 2001; Badyaev 2002; Gorman and Nager 2004). Control males did not differ in survival to fledging from the rest of the chicks in this study; however, fledging in poorer condition may have reduced their future survival prospects (e.g. Hochachka and Smith 1991; GebhardtHenrich and Richner 1998).

In summary, our results suggest that in a sexually sizedimorphic species, the herring gull, the sexes differ in their ability to offset costs posed by hatching asynchrony with females having an advantage in hatching last. Such sex differences in vulnerability to environment during embryonic development can have important implications for sex allocation within clutches (Uller 2006). They could help explain why mothers often produce more offspring of the less vulnerable sex towards the end of the laying sequence, especially under unfavourable conditions (e.g. Nager et al. 1999; Ležalová et al. 2005). However, the mechanism(s) underlying these differences between the sexes remain to be established.
Acknowledgements We thank Pat Monaghan for her support and contribution at various stages of this work. Kate Griffiths, Nanette Verboven, Sin-Yeon Kim, Liliana D'Alba and the Cumbria Wildlife Trust provided invaluable help during fieldwork, and Kate Griffiths and Aileen Adam sexed the birds. We are grateful to Jan Lindström, Neil Metcalfe and three anonymous referees for helpful comments on a previous version of the manuscript. The study was carried out under a licence from English Nature; blood samples were taken under a licence from the UK Home Office. M.I.B. was funded by a University of Glasgow Postgraduate Scholarship and the ORS scheme.

\section{References}

Arendt JD (1997) Adaptive intrinsic growth rates: an integration across taxa. Q Rev Biol 72:149-177

Arnold JM, Hatch JJ, Nisbet ICT (2006) Effects of egg size, parental quality and hatch-date on growth and survival of Common Tern Sterna hirundo chicks. Ibis 148:98-105

Badyaev AV (2002) Growing apart: an ontogenetic perspective on the evolution of sexual size dimorphism. Trends Ecol Evol 17:369378

Balaban M, Hill J (1971) Effects of thyroxine level and temperature manipulations upon the hatching of chicken embryos (Gallus domesticus). Dev Psychobiol 4:17-35

Bize P, Roulin A, Richner H (2002) Covariation between egg size and rearing condition determines offspring quality: an experiment with the alpine swift. Oecologia 132:231-234

Blanco G, Martinez-Padilla J, Davila JA, Serrano D, Viñuela J (2003) First evidence of sex differences in the duration of avian embryonic period: consequences for sibling competition in sexually dimorphic birds. Behav Ecol 14:702-706

Bogdanova MI, Nager RG, Monaghan P (2006) Does parental age affect offspring performance through differences in egg quality? Funct Ecol 20:132-141

Bogdanova MI, Nager RG, Monaghan P (2007) Age of the incubating parents affects nestling survival: an experimental study of the herring gull Larus argentatus. J Avian Biol 38:83-93

Brua RB (ed) (2002) Parent-embryo interactions. In: Avian incubation. Behaviour, environment, and evolution. Oxford University Press, New York, pp 88-99

Burke WH (1992) Sex-differences in incubation length and hatching weights of broiler chicks. Poultry Sci 71:1933-1938

Christians JK (2002) Avian egg size: variation within species and inflexibility within individuals. Biol Rev 77:1-26

Clutton-Brock TH (1991) The evolution of parental care. Princeton University Press, Princeton New Jersey

Cook MI, Monaghan P (2004) Sex differences in embryo development periods and effects on avian hatching patterns. Behav Ecol 15:205-209

Coulson JC, Thomas CS, Butterfield JEL, Duncan N, Monaghan P, Shedden C (1983) The use of head and bill length to sex live gulls Laridae. Ibis 125:549-557

Cramp S (ed) (1985) Herring gull. In: Handbook of the birds of the Western Palearctic, vol 4. Oxford University Press, Oxford UK, pp 815-837

Drent RH (1970) Functional aspects of incubation in the Herring Gull. Behaviour Suppl 17:1-132

Dunnington EA, Siegel PB, McNabb FMA (1993) Hatching time, body weight, and thyroid hormones in male and female chicks from lines selected for high or low juvenile body weight. Poultry Sci 72:1998-2000

Eising CM, Eikenaar C, Schwabl H, Groothuis TGG (2001) Maternal androgens in black-headed gull (Larus ridibundus) eggs: con- 
sequences for chick development. Proc Roy Soc Lond B 268:839-846

Gasparini J, Roulin A, Gill VA, Hatch SA, Boulinier T (2006) Kittiwakes strategically reduce investment in replacement clutches. Proc Roy Soc Lond B 273:1551-1554

Gebhardt-Henrich S, Richner H (1998) Causes of growth variation and its consequences for fitness. In: Starck JM, Ricklefs RE (eds) Avian growth and development. Oxford University Press, Oxford, pp 324-339

Gorman HE, Nager RG (2004) Prenatal developmental conditions have long-term effects on offspring fecundity. Proc Roy Soc Lond B 271:1923-1928

Göth A, Booth DT (2005) Temperature-dependent sex ratio in a bird. Biol Letters 1:31-33

Griffiths R (1992) Sex-biased mortality in the Lesser Black-backed Gull Larus fuscus during the nestling stage. Ibis 134:237-244

Griffiths R, Double MC, Orr K, Dawson RJG (1998) A DNA test to sex most birds. Mol Ecol 7:1071-1075

Groothuis TGG, Müller W, von Engelhardt N, Carere C, Eising C (2005) Maternal hormones as a tool to adjust offspring phenotype in avian species. Neurosci Biobehav Rev 29:329-352

Hébert PN, Barclay RMR (1986) Asynchronous and synchronous hatching: effect on early growth and survivorship of Herring Gull, Larus argentatus, chicks. Can J Zool 64:2357-2362

Hillström L, Kilpi M, Lindström K (2000) Is asynchronous hatching adaptive in Herring Gulls (Larus argentatus)? Behav Ecol Sociobiol 47:304-311

Hochachka W, Smith JNM (1991) Determinants and consequences of nestling condition in song sparrows. J Anim Ecol 60:995-1008

Lee SC, Evans RM, Bugden SC (1993) Benign neglect of terminal eggs in herring gulls. Condor 95:507-514

Ležalová R, Tkadlec E, Oborník M, Šimek J, Honza M (2005) Should males come first? The relationship between offspring hatching order and sex in the black-headed gull Larus ridibundus. J Avian Biol 36:478-483

Lindström J (1999) Early development and fitness in birds and mammals. Trends Ecol Evol 14:343-348

Lipar JL, Ketterson ED (2000) Maternally derived yolk testosterone enhances the development of the hatching muscle in the redwinged blackbird Agelaius phoeniceus. Proc Roy Soc Lond B 267:2005-2010

Metcalfe NB, Monaghan P (2001) Compensation for a bad start: grow now, pay later? Trends Ecol Evol 16:254-260

Mock DW, Parker GA (1997) The evolution of sibling rivalry. Oxford University Press, New York

Muck C, Nager RG (2006) The effect of laying and hatching order on the timing and asynchrony of hatching. Anim Behav 71:885-892

Nager RG, Monaghan P, Griffiths R, Houston DC, Dawson R (1999) Experimental demonstration that offspring sex ratio varies with maternal condition. Proc Natl Acad Sci U S A 96:570-573

Nager RG, Monaghan P, Houston DC (2000a) Within-clutch tradeoffs between the number and quality of eggs: experimental manipulations in gulls. Ecology 81:1339-1350

Nager RG, Monaghan P, Houston DC, Genovart M (2000b) Parental condition, brood sex ratio and differential young survival: an experimental study in gulls (Larus fuscus). Behav Ecol Sociobiol 48:452-457

Nilsson JÅ, Persson I (2004) Postnatal effects of incubation length in mallard and pheasant chicks. Oikos 105:588-594
Parsons J (1970) Relationship between egg size and post-hatching chick mortality in the Herring Gull (Larus argentatus). Nature 228:1221-1222

Parsons J (1975) Asynchronous hatching and chick mortality in the Herring Gull Larus argentatus. Ibis 117:517-520

Pérez C, Velando A, Domínguez J (2006) Parental food conditions affect sex-specific embryo mortality in the yellow-legged gull (Larus michahellis). J Ornithol 147:513-519

Persson I, Andersson G (1999) Intraclutch hatch synchronisation in pheasants and mallard ducks. Ethology 105:1087-1096

Quinn GP, Keough MJ (2003) Experimental design and data analysis for biologists. Cambridge University Press, Cambridge

Ricklefs RE, Starck JM (1998) Embryonic growth and development. In: Starck JM, Ricklefs RE (eds) Avian growth and development. Oxford University Press, New York, pp 31-58

Rutkowska J, Cichoń M (2002) Maternal investment during egg laying and offspring sex: an experimental study of zebra finches. Anim Behav 64:817-822

Rytkönen S (2002) Nest defence in great tits Parus major: support for parental investment theory. Behav Ecol Sociobiol 52:379384

Salomons HM, Müller W, Dijkstra C, Eising CM, Verhulst S (2006) No sexual differences in embryonic period in jackdaws Corvus monedulla and black-headed gulls Larus ridibundus. J Avian Biol 37:19-22

SAS Institute (2001) SAS Software, Release 8.2. SAS Institute, Cary, USA

Schew WA, Ricklefs RE (1998) Developmental plasticity. In: Starck JM, Ricklefs RE (eds) Avian growth and development. Oxford University Press, New York, pp 288-304

Sheldon BC, Merilä J, Lindgren G, Ellegren H (1998) Gender and environmental sensitivity in nestling collared flycatchers. Ecology 79:1939-1948

SPSS Inc (2003) SPSS for Windows, Release 12.0. SPSS, Chicago, USA

Stoleson SH, Beissinger SR (1995) Hatching asynchrony and the onset of incubation in birds, revisited: when is the critical period? In: Power D (ed) Current ornithology. vol. 12. Plenum, New York, pp 191-270

Uller T (2006) Sex-specific sibling interactions and offspring fitness in vertebrates: patterns and implications for maternal sex ratios. Biol Rev 81:207-217

Vince MA (1969) Embryonic communication, respiration and the synchronisation of hatching. In: Hinde RA (ed) Bird vocalizations. Cambridge University Press, Cambridge, pp 233-260

Vleck CM, Bucher TL (1998) Energy metabolism, gas exchange, and ventilation. In: Starck JM, Ricklefs RE (eds) Avian growth and development. Oxford University Press, New York, pp 89-116

Webb DR (1987) Thermal tolerance of avian embryos: a review. Condor 89:874-898

Williams TD (1994) Intra-specific variation in egg size and egg composition in birds: effects on offspring fitness. Biol Rev 68:35-59

Winkler DW, Allen PE (1996) The seasonal decline in tree swallow clutch size: physiological constraint or strategic adjustment? Ecology 77:922-932

Woolf NK, Bixby JL, Capranica RR (1976) Prenatal experience and avian development: brief auditory stimulation accelerates the hatching of Japanese quail. Science 194:959-960 